\title{
SEMIGROUPS WITH INVARIANT RADON MEASURES
}

\author{
CHANDRA GOWRISANKARAN
}

\begin{abstract}
Let $S$ be a commutative semigroup which is a topological space such that the translations are both continuous and open maps. The main result states that if (1) either $S$ is Suslin such that there is at least one point of continuity for the semigroup mapping $S \times S \rightarrow S$ or $S$ is polish and (2) $\exists$ a nontrivial Radon measure on $S$ such that $\mu(V)=\mu(x+V)$ for $V$ open $\subset S$ and $x \in S$, then $S$ can be embedded as an open subsemigroup of a locally compact group. It is also shown that if $S$ is polish and a cancellation semigroup then $S$ can be embedded as an open subsemigroup of a group.
\end{abstract}

In [6], R. Rigelhof proved that if $S$ is a locally compact abelian semigroup such that the translations are both continuous and open and $\exists$ a Radon measure $\mu$ such that $\forall x \in S, \forall$ nonvoid open set $V \subset S$, $\mu(x+V)=\mu(V)>0$, then $S$ can be embedded as an open subsemigroup of a locally compact group. In the context of our main result (stated in the abstract) we first show that $\forall$ nonvoid open set $V \subset S, \mu(V)>0$. We then follow the methods of [6] to get a topology on the abelian group $G$ generated by $S$. At this stage, we cannot conclude that $G$ is a topological group. We proceed as follows to show that $G$ is a locally compact space. We first construct a translation invariant Radon measure $\lambda$ on $G$ which extends the measure $\mu$ on $S$. Then, modifying an idea of D. Montgomery [5] we conclude that the images of certain 'good' subsets of $G$ under inversion are universally measurable, enabling us to get a compact set $K \subset G$ with the property $\lambda(K)>0$ and $-K$ is compact. Then, as in [3], we show that $G$ is locally compact.

A small modification of the above method (Lemmas 2 and 6) and a result of $\mathrm{Wu}$ [9] yield an embedding theorem (Theorem 2) for polish commutative cancellation semigroups in which translations are also open maps. This embedding theorem gives an alternative and somewhat simpler proof of the main result for the case of polish semigroups.

In the sequel $S$ will be a semigroup and $\mu$ a Radon measure on it satisfying the conditions stated in the main result.

Received by the editors May 16, 1972 and, in revised form, August 24, 1972.

AMS (MOS) subject classifications (1969). Primary 2205; Secondary 2875.

Key words and phrases. Polish, Suslin, semigroup, invariant measure, topological group, Haar measure, Radon measure.

(c) American Mathematical Society 1973 
LEMMA 1. For every nonvoid open subset $V \subset S, \mu(V)>0$ and hence $S$ is a cancellation semigroup. Further for every Borel subset $B \subset S, x+B$ is Borel and $\mu(x+B)=\mu(B)$.

Proof. Suppose $\exists$ a nonvoid open subset $V$ such that $\mu(V)=0$. Let $K$ be an arbitrary compact subset of $S$. Then, the open set $K+V$ is the union of open sets $x+V, x \in K$, of $\mu$ measure zero and so $\mu(K+V)=0$. Let $x \in V$ and $W=\{y \in S: x+y \in K+V\}$. Then $W$ is open and $\mu(W)=$ $\mu(x+W) \leqq \mu(K+V)=0$. But $K \subset W$ and hence $\mu(K)=0$. This is clearly a contradiction. With the observation that a Radon measure is by definition locally finite, we can deduce exactly as in [6, p. 175], that $S$ is a cancellation semigroup. Now, for every $x \in S, y \rightarrow x+y$ is a homeomorphism of $S$ onto the open subset $x+S$. It follows immediately that $x+B$ is a Borel set for every Borel set $B \subset S$. Finally, $\mu(B)=\mu(x+B)$ is an easy consequence of the outer regularity of $\mu$. The lemma is proved.

Let us now suppose that $G$ is the abelian group generated by $S$; i.e. consider $S \times S$ and define an equivalence relation $(x, y) \sim\left(x^{\prime}, y^{\prime}\right)$ is $x+y^{\prime}=x^{\prime}+y$. Then $G$ is the set of equivalence classes and $S$ is identified as a subsemigroup by the monomorphism $x \rightarrow$ the class containing $(x+y, y)$. Clearly, every element in $G$ can be written in the form $x-y$ where $x \in S, y \in S$. Now, exactly as in [6, p. 174] we deduce the following lemma.

LeMma 2. Let $x_{0} \in S$, and $N$ the filter of all neighborhoods of $x_{0} \in S$. Let $N(x)$ be the filter generated on $G$ by $\left\{V+x-x_{0}: V \in N\right\} \forall x \in G$. Then there is a Hausdorff topology on $G$ which is locally Suslin, such that $N(x)$ is precisely the filter of all neighborhoods of $x \in G$. Further, $S$ is an open subset of $G$ and the translations $x \rightarrow x+y$ are homeomorphisms of $G$.

LeMma 3. The mapping $(x, y) \rightarrow x+y$ is continuous from $G \times G \rightarrow G$.

Proof. If the space $S$ is polish, then $(x, y) \rightarrow x+y$ is of Baire class 1 and therefore has at least one point of continuity [4]. But $S \subset G$ and $S \times S \subset G \times G$ are both open and hence this point is also a point of continuity of $(x, y) \rightarrow x+y$ on $G \times G$. The rest of the proof is standard. Obviously the same conclusion can be derived by starting with the assumption that there is a point of continuity on $S \times S$ for the mapping $(x, y) \rightarrow x+y$. The proof is complete.

Lemma 4. Let $X \subset G$ be such that with the induced topology $X$ is a Suslin space. If $-X=\{-x: x \in X\}$, then for every point of $G$ there is an open neighborhood $V$ such that $-X \cap V$ is Suslin. In particular $-X$ is $v$ measurable for every positive Radon measure $v$ on $G$. 
Proof. Let $y \in G$ and let $V$ be an open neighborhood of $y$ such that $V$ is a Suslin space (Lemma 2). By Lemma 3, the set

$$
M=\{(x, z) \in G \times G: x+z=0\}
$$

is closed. Hence, $M \cap(V \times X)$, a closed subspace of $V \times X$, is a Suslin space. But $V \cap-X$ is precisely the (one-one, continuous) projection of $M \cap(V \times X)$ and is hence a Suslin space. Hence, $-X$ is locally $v$-measurable and it follows that $-X$ is $v$-measurable [8, Part I]. The lemma is proved.

LEMma 5. There exists an invariant Radon measure $\lambda$ on $G$ which extends the measure $\mu$ on $S$.

Proof. Let $x_{0}$ be fixed in $S$ and $V$ a Suslin neighborhood of $x_{0}$ in $S$ such that $0<\mu(V)<\infty$. Let $x \in G$ and $V_{x}=V-x_{0}+x$. Let $\lambda_{x}$ be the Radon measure on $V_{x}$ which is the image of $\mu$ restricted to $V$ under the homeomorphism $y \rightarrow y-x_{0}+x$ of $V \rightarrow V_{x}$. We shall see that measures $\lambda_{x}$ defined on $V_{x}, x \in G$, are consistent. Suppose, $B$ is a Borel set contained in $V_{x} \cap V_{y}$ for $x \neq y, x, y \in G$. Suppose $B_{1}=\left\{z+x_{0}-x: z \in B\right\}$ and $B_{2}=$ $\left\{z+x_{0}-y: z \in B\right\}$. Then, $B_{1}$ and $B_{2}$ are Borel subsets of $V$ and $B_{1}+(x-y)=$ $B_{2}$ and $\lambda_{x}(B)=\mu\left(B_{1}\right)$ and $\lambda_{y}(B)=\mu\left(B_{2}\right)$. But since $x-y=x^{\prime}-y^{\prime}$, where $x^{\prime}, y^{\prime} \in S$, we get from Lemma 1 ,

$$
\begin{aligned}
\mu\left(B_{2}\right) & =\mu\left(B_{1}+x^{\prime}-y^{\prime}\right)=\mu\left(B_{1}+x^{\prime}-y^{\prime}+y^{\prime}\right) \\
& =\mu\left(B_{1}+x^{\prime}\right)=\mu\left(B_{1}\right) .
\end{aligned}
$$

Hence these Radon measures are consistent and we can glue them up to get a unique Radon measure $\lambda$ on $G$ [8]. It is quite easy to verify that $\lambda$ restricted to $S$ is exactly $\mu$. It remains to show that $\lambda$ is invariant. Let $z \in G$. If $B$ is a Borel set $\subset$ some $V_{x}$, then $\lambda(B)=\mu\left(B-x+x_{0}\right)$ and $z+B \subset V_{x+z}$ and $\lambda(z+B)=\mu\left(B+z-z+x+x_{0}\right)=\lambda(B)$. If $K$ is any compact set $\subset G, K \subset \bigcup_{k=1}^{n} V_{x_{k}}$ and $K$ can be written as a disjoint union of Borel sets $B_{1}, B_{2}, \cdots, B_{m}$ such that each $B_{i}$ is contained in some $x_{i}+V$. Now, $\lambda(K)=\sum \lambda\left(B_{i}\right)=\sum \lambda\left(z+B_{i}\right)=\lambda\left[U\left(z+B_{i}\right)\right]=\lambda(z+K)$. The translation invariance follows from the inner regularity of $\lambda$. The proof is complete.

LEMMA 6. $G$ can be covered by a countable number of open sets $V_{n}$ such that, for every $n$, (1) $V_{n}$ with the induced topology is Suslin and (2) $\lambda\left(V_{n}\right)<$ $+\infty$.

Proof. Let $\left\{W_{n}\right\}$ be a covering of $S$ by open sets of finite $\lambda$-measure.

$$
G=S-S=\bigcup_{n=1}^{\infty} W_{n}-\bigcup_{m=1}^{\infty} W_{m}=\bigcup_{n=1}^{\infty} \bigcup_{m=1}^{\infty}\left(W_{n}-W_{m}\right)
$$

To preve the lemma, it is enough to show that $W_{n}-W_{m}$ can be covered by a countable number of Suslin open sets of finite measure. Let $\left\{\alpha_{k}\right\}_{k=1}^{\infty}$ be a dense subset of $W_{m}$. We assert that $W_{n}-W_{m}=\bigcup_{k=1}^{\infty}\left(W_{m}-\alpha_{k}\right)$. Clearly $W_{n}-W_{m}$ contains the set on the right side. Conversely, suppose 
$z \in W_{n}-W_{m}$, say $z=x-y$ where $x \in W_{n}, y \in W_{m}$. Then $y$ is in $W_{n}-z$ and hence there is a neighborhood $U$ of $y$ such that $U$ is contained in $W_{n}-z$ and $U$ is contained in $W_{m}$. Hence we can find an $\alpha_{k}$ in $U$ such that $\alpha_{k}$ is in $W_{n}-z$, that is $z$ is in $W_{n}-\alpha_{k}$. Now, by the choice, each $W_{n}-\alpha_{k}$ is Suslin and $\lambda\left(W_{n}-\alpha_{k}\right)=\lambda\left(W_{n}\right)<+\infty$. The lemma is proved.

COROllary 1. $\exists$ at least one Suslin open set $V \subset G$ such that $\lambda(-V)>0$.

Proof. Consider the countable family $\left\{V_{n}\right\}$ given by the lemma. Then, $-V_{n}$ is $\lambda$-measurable (Lemma 4) and $G=\bigcup_{n=1}^{\infty}-V_{n}$. This completes the proof.

Corollary 2. $\exists$ at least one compact set $K \subset G$ such that $\lambda(K)>0$ and - $K$ is compact.

Proof. Let $V$ be a Suslin open set of $G$ such that $\lambda(-V)>0$. Since every Borel set $B \subset V$ is Suslin, by Lemma $4,-B \subset-V$ is $\lambda$-measurable. Again, since $V$ is Suslin, this implies that the mapping $-x$ of $-V \rightarrow V$ is $\lambda$-Lusin measurable [8, Part I]. Hence $\exists$ a compact subset $K$ of $-V$ of $\lambda$ measure $>0$ such that $x \rightarrow-x$ is continuous when restricted to $K$. This implies in particular that $-K$ is compact, completing the proof.

THEOREM 1. G is a locally compact topological group and $\lambda$ is a Haar measure on $G$. Consequently $S$ is locally compact and $\mu$ is the restriction to $S$ of a Haar measure on $G$.

Proof. Let $K \subset G$ compact such that $-K$ is compact and $\lambda(K)>0$. Let $0<\varepsilon<\lambda(K)$ and $W$ an open set $\supset K$ such that $\lambda(W)<\lambda(K)+\varepsilon$. We can choose a neighborhood $V$ of 0 such that $V+K \subset W$. Hence, $\forall x \in V$, $x+K \subset W$ and $\lambda(x+K)=\lambda(K)>\lambda(W)-\varepsilon$. It follows that $\lambda(x+K \Delta K)<2 \varepsilon$.

Now

$$
\begin{aligned}
K+(-K) & \supset\{x \in G:(x+K) \cap K \neq \varnothing\} \\
& \supset\{x \in G: \lambda(x+K \Delta K)<2 \varepsilon\} \supset V .
\end{aligned}
$$

But, again by Lemma $3, K+(-K)$ is compact and we get that $G$ is locally compact. Now this implies that $G$ is a topological group [1]. The proof is complete.

COROLlary. Let $G$ be a locally compact Hausdorff group and $S$ a subsemigroup such that with the induced topology $S$ is a Suslin space and the Haar measure of $S$ is $>0$. Then $S$ is locally compact.

REMARK 1. $G$ (as in the theorem) is the continuous image of $S \times S$ under the mapping $(x, y) \rightarrow x-y$ and is hence Suslin; also, $G$ is locally compact and we deduce that $G$ is necessarily polish.

REMARK 2. It is fairly straightforward to see that the topology constructed here is the same as the quotient topology given by the mapping $S \times S \rightarrow G$. 
A slightly different but somewhat simpler proof of Theorem 1 can be given in the case of polish semigroups. The proof is based on Lemma 5 $[3$, p. 383] and the following result which is itself of independent interest ([2], [7]).

THEOREM 2. Let $S$ be a commutative cancellation semigroup which is a polish space such that $x \rightarrow x+y$ is both open and continuous for every $y \in S$. Then $S$ can be algebraically and topologically embedded as an open subsemigroup of a metrizable Lusin group $G$.

Proof. Let $G$ be the group generated by $S$ and provide the locally polish topology on $G$, as in Lemma 2, such that $S$ is identified as an open subsemigroup of $G$. It follows immediately that $G$ is a regular space and is of second category. The proof of Lemma 6 shows that $G$ is a countable union of polish open sets $V_{n} \subset G$. Clearly, $\left\{\beta_{n}, n=1,2, \cdots\right\}$, where $\beta_{n}$ is a countable base for the open sets of $V_{n}$, for each $n$, is a countable base for the topology of $G$. It follows by a theorem of $\mathrm{Wu}$ [9] that $G$ is a topological group. It is easy to deduce the other topological properties of $G$, completing the proof.

REMARK. Using the results in [2 ${ }^{\text {bis }}$, pp. 314,207$], G$ can be shown to be a polish space.

\section{BIBLIOGRAPHY}

1. R. Ellis, A note on the continuity of the inverse, Proc. Amer. Math. Soc. 8 (1957), 372-373. MR 18, 745.

2. B. R. Gelbaum, G. K. Kalisch and J. M. H. Olmstead, On the embedding of topological semigroups and integral domains, Proc. Amer. Math. Soc. 2 (1951), 807-821. MR 13, 206.

$2^{\text {bis }}$. J. Dungundji, Topology, Allyn \& Bacon, Boston, Mass., 1966. MR 33 \#1824.

3. C. Gowrisankaran, Radon measures on groups, Proc. Amer. Math. Soc. 25 (1970), 381-384. MR 41 \#384.

4. K. Kuratowski, Topology. Vol. 1, rev. ed., Academic Press, New York; PWN, Warsaw, 1966. MR 36 \#840.

5. D. Montgomery, Continuity in topological groups, Bull. Amer. Math. Soc. 42 (1936), 879-882.

6. R. Rigelhof, Invariant measures on locally compact semigroups, Proc. Amer. Math. Soc. 28 (1971), 173-176. MR 43 \#3424.

7. N. J. Rothman, Embedding of topological semigroups, Math. Ann. 139 (1960), 197-203. MR 22 \#6871.

8. L. Schwartz, Radon measures on general topological spaces, Tata Institute of Fundamental Research Monographs (to appear).

9. Ta-Sun Wu, Continuity in topological groups, Proc. Amer. Math. Soc. 13 (1962), 452-453. MR 25 \#1234.

Department of Mathematics, McGill University, Montreal, Quebec, Canada 